\title{
Color-Matching Liquid Crystal Display using a Lenticular Lens Array and RGB Light Sources
}

\author{
Hwa Joon Jeon', Gyeungju Park², Jin Seog Gwag', Jong Hoon Lee', and Jin Hyuk Kwon* \\ ${ }^{1}$ Department of Physics, Yeungnam University, Gyeongsan 712-749, Korea \\ ${ }^{2}$ Department of Optometry, Sunlin College, Pohang 791-912, Korea
}

(Received May 22, 2014 : revised July 28, 2014 : accepted July 28, 2014)

\begin{abstract}
A direct-lit color-matching liquid crystal display using a lenticular lens array with grouped lens elements that image linear RGB light sources on the RGB subpixels of a color filter to enhance transmittance is designed, simulated, and fabricated. The RGB LED linear light sources were fabricated using small RGB LEDs in a linear array arrangement, and the lenticular lens array consisted of eight units of the same structure with a gap of $2.19 \mathrm{~mm}$. The optical transmittance of the liquid crystal panel was improved by as much as $240 \%$ due to the color matching.
\end{abstract}

Keywords : LCD, High efficiency, Color matching

OCIS codes : (230.0230) Optical devices; (080.2740) Geometric optical design; (220.4000) Microstructure fabrication; (230.3990) Micro-optical devices

\section{INTRODUCTION}

The major issues of future liquid crystal displays (LCDs) will be energy efficiency and image resolution, as LCDs become larger and consume more energy in proportion to size. The resolution issue has been already addressed very well by employing the ultra-high definition (UHD) format that has twice the pixel density of the current full high definition (FHD) format, and it seems that there remain no serious technical problems [1]. However, concerning energy efficiency there is no clear solution so far, although there have been many efforts to increase efficiency. The typical light transmittance of the LCD panel remains as low as about $5 \%$, mainly due to absorption in the polarizer and color filter (CF) of the LCD panel. The effort to improve energy efficiency includes three approaches. The first is developing a polarized backlight that illuminates the liquid crystal panel (LCP) with polarized light, to avoid absorption by the bottom polarizer [2-4]. The second is using field sequential color (FSC), a time-domain multiplexing technology to enhance the transmittance of the LCP by removing the color filter [5]. This technology is especially attractive because it can enhance the light energy efficiency as well as the removal of subpixels of the LCP and color filter. Despite many efforts, however, it turned out that the color breakup problem is very hard to overcome, and most technologies to reduce the color breakup in FSC images have not been successful [6]. The third approach to enhance energy efficiency is using a color-matching technique that utilizes a three-color backlight to match the RGB subpixels of the color filter [7, 8]. Yamada et. al proposed a color-filterless LCD employing an edge-lit backlight that belongs to the spatial-domain multiplexing technology [9]. They utilized a blazed grating and lenticular lens array (LLA) to separate the red, green, and blue (RGB) colors from the white backlight and then focus the RGB light onto the corresponding RGB subpixels of an LCD panel from which the $\mathrm{CF}$ had been removed. Although they demonstrated a prototype LCD without a color filter, an increase of transmittance or light energy efficiency was not reported. It seems that the complicated optical structure comprised of the blazed grating and the lenticular lens array induces additional loss of light such as zeroth-order diffracted light, besides the complexity of the backlight system.

\footnotetext{
*Corresponding author: jhkwon@ynu.ac.kr

Color versions of one or more of the figures in this paper are available online.
} 


\section{OPTICAL DESIGN, SIMULATION, AND FABRICATION}

We propose a new direct-lit color-matching LCD scheme to enhance the light energy efficiency by employing a specially designed lenticular lens array and RGB linear light sources, as shown in Fig. 1. Although many current LCDs larger than 40 inches employ the edge-lit backlight system, the trend is changing back to the direct-lit backlight system because of its higher image quality and lower cost. The light sources are placed as linear arrays of RGB light-emitting diodes (LEDs) at a distance $a$ from the LLA. The LLA may be placed on the surface of the rear glass panel of thickness $T_{1}$. The thickness of the liquid crystal layer is about $4 \mu \mathrm{m}$, so the distance $b$ between the LLA and the CF is given by the sum of $T_{1}$ and the LC thickness. The simple lens equation holds among the LLA, RGB LEDs, and the color filter as follows:

$$
\frac{1}{a}+\frac{n}{b}=\frac{n}{f}
$$

where $f$ and $n$ are the focal length of the lens element of the LLA and the refractive index of the LLA respectively. The direct-lit color-matching LCD is composed of several units including the RGB LED, LLA, and CF to form a large LCD system. The adjacent LLA units have a separation gap $g$ between them, as shown in Fig. 1. From the optical arrangement of Fig. 1 that satisfies Eq. (1), it is possible that all RGB rays from the RGB LEDs entering the corresponding LLA unit fall onto the respective RGB color filter. The color-matching illumination can enhance the $\mathrm{CF}$ transmittance by as much as about $300 \%$ theoretically because the color-matched transmittance reaches about $90 \%$, compared to the low transmittance of about 30\% when white light is used, and so the energy efficiency of the

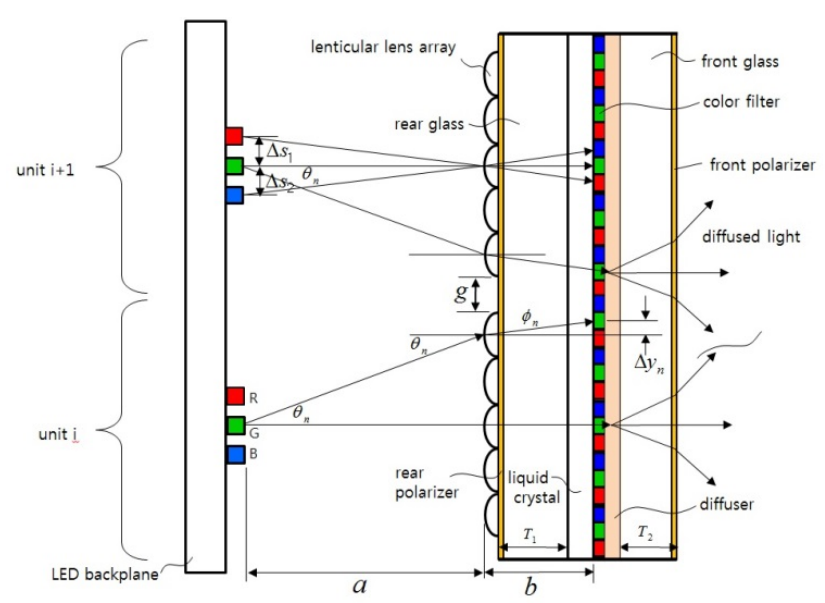

FIG. 1. A schematic of a direct-lit color-matching LCD that is composed of several units of optical structure, consisting of an RGB LED array, a lenticular lens array, and a color filter.
LCD will be increased dramatically. However, there is some light loss due to rays from the LEDs falling outside the LLA of the corresponding unit. The separation gap between the adjacent LLA units is required to distribute equally the RGB light from the RGB LEDs into the RGB color filter subpixels. The required separation gap is

$$
g=2 b \tan \phi_{n}
$$

The offset distance $\Delta y_{n}$ between the vertical positions of the $n$th LLA lens element and the $n$th CF subpixel is given by the following equation, due to refraction at the lens elements:

$$
\begin{aligned}
& \Delta y_{n}=b \tan \phi_{n}, \\
& \tan \theta_{n}=n P / a,
\end{aligned}
$$

where $P$ is the pitch of the color filter subpixels along the vertical direction. The separation between the RGB LEDs should be adjusted according to the following equation, due to the dispersion among the red, green, and blue wavelengths:

$$
\Delta s_{2}=\frac{n\left(\lambda_{r}\right)}{n\left(\lambda_{b}\right)} \Delta s_{1},
$$

where $\Delta s_{1}$ and $\Delta s_{2}$ are the separation distances between the green and blue LEDs and the green and red LEDs respectively.

We performed a Monte Carlo optical simulation to check the performance of the direct-lit color-matching LCD system. The panel size was $120 \times 100 \mathrm{~mm}^{2}$ and the parameters were $a=20 \mathrm{~mm}, b=0.825 \mathrm{~mm}$, and $n=1.5$. The spacing between the red, green, and blue LEDs was $1.025 \mathrm{~mm}$. The LEDs used were very small, $1.8 \times 1 \times 0.4 \mathrm{~mm}^{3}$, to make the spacing between adjacent LEDs as small as possible, as color nonuniformity becomes smaller with smaller LED spacing. The height and width of the LLA were $15.7 \mu \mathrm{m}$ and $0.541 \mathrm{~mm}$ respectively, with 27 lens elements per unit. The separation gap between adjacent LLA units was $2.19 \mathrm{~mm}$. The $\mathrm{CF}$ period was $0.5415 \mathrm{~mm}$, a typical value for 50-inch LCDs. There were eight units for the prototype width of $120 \mathrm{~mm}$. A diffuser sheet with a Gaussian scattering distribution of angular spread 20 degrees was assumed to be placed between the CF and front glass. The liquid crystal layer thickness of $4 \mu \mathrm{m}$ was not included because it has little influence on the performance of the optical transmittance.

The simulated results for the enhancement factors for luminance and illuminance were $195 \%$ and $146 \%$, respectively. The view angles were 103.5 and 76.5 degrees along the horizontal and vertical directions respectively. The color nonuniformity was CIE $\Delta x=0.019$ and CIE $\Delta y=0.01$. The 


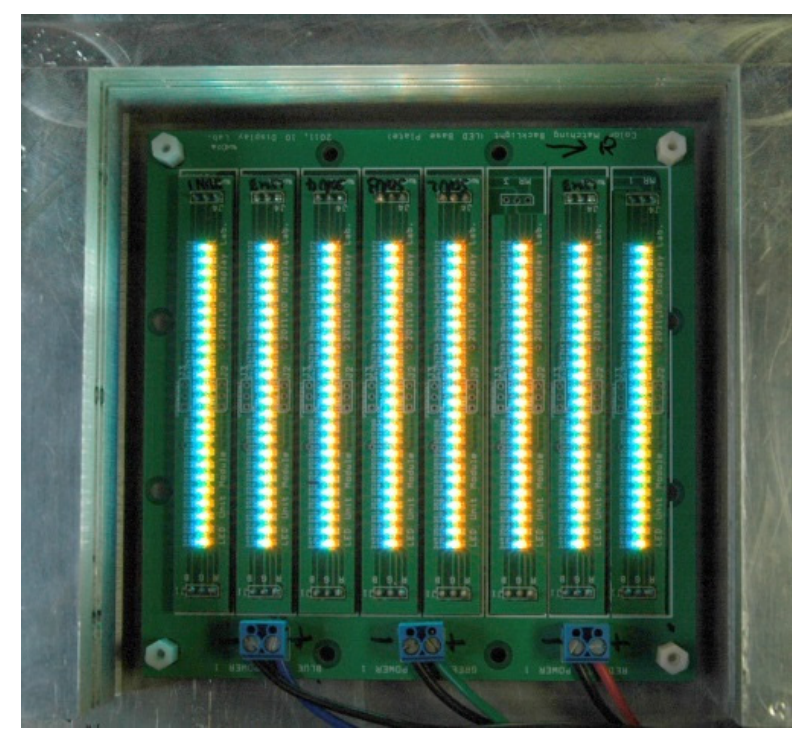

FIG. 2. The RGB LED array module used as the RGB light sources.

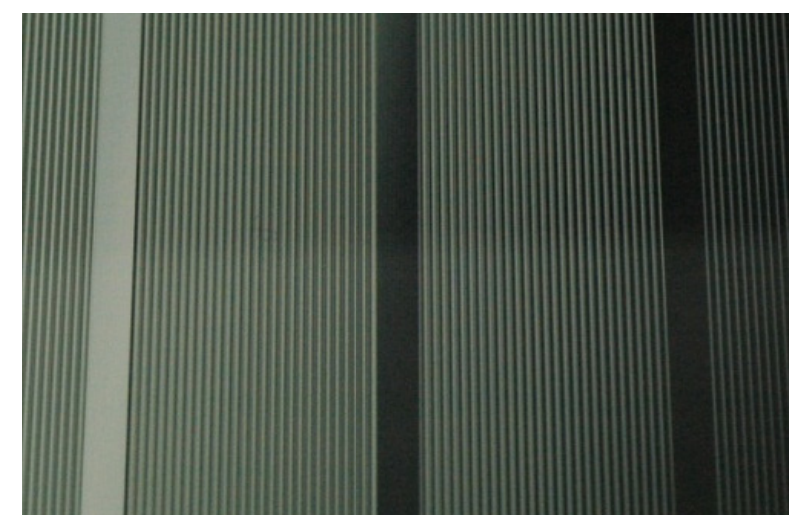

FIG. 3. Photo of the LLA sheet with separation gap of $2.19 \mathrm{~mm}$ between the adjacent units. Each unit has 27 lenticular lens elements.

uniformities of the illuminance and spatial luminance were $92.5 \%$ and $82.9 \%$ respectively. The results of the simulation showed that the direct-lit color-matching LCD system has the potentialto increase the energy efficiency of an LCD while maintaining its optical characteristics.

A prototype direct-lit color-matching LCD system was constructed by fabricating and assembling the RGB LED linear arrays, the LLA sheet with 8 units, and the CF according to the simulation model. Figure 2 shows the RGB LED array module with RGB light beams emitted by the RGB LEDs. The peak wavelength and power for each of the RGB LEDs were $650 \mathrm{~nm}, 520 \mathrm{~nm}$, and $470 \mathrm{~nm}$ and $3.6 \mathrm{~lm}, 9 \mathrm{~lm}$, and $1.8 \mathrm{~lm}$ respectively. The separations between the adjacent red, green and blue LEDs were chosen to be $1.03 \mathrm{~mm}$ because the width of the CF subpixels was sufficient, compared to the width of the RGB color lines formed on the $\mathrm{CF}$ subpixels. Figure 3 shows a magnified portion of the fabricated LLA sheet. The height,

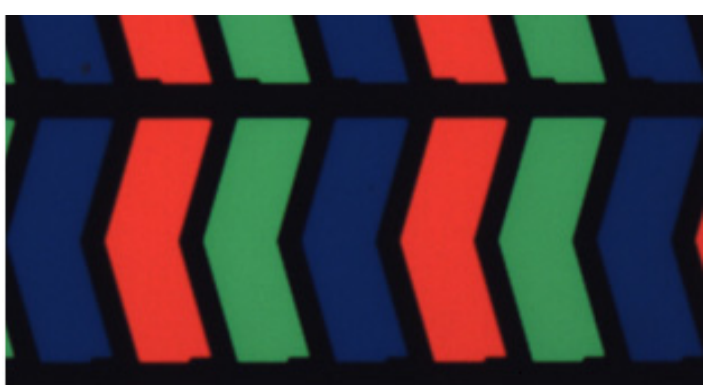

(a)

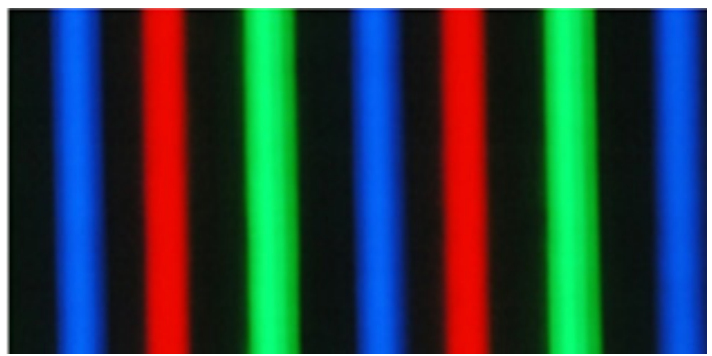

(b)

FIG. 4. The RGB color beams (b) formed at the CF by the lenticular lens array and the RGB LED arrays. It is seen that each RGB color beam matches its RGB color filter subpixel (a).

radius and width of each lenticular lens were $15.7 \mu \mathrm{m}, 1.7 \mu \mathrm{m}$, and $531 \mu \mathrm{m}$ respectively, and the separation gap between the adjacent units of the LLA was $2.19 \mathrm{~mm}$. The LLA sheet was made via the roll transfer process from a rolltype base moldmade using a photoresist thermal reflow procedure [10]. The top and bottom panels of Fig. 4 show the color filter used in the prototype; it had a period of $541.5 \mu \mathrm{m}$ with a chevron pattern. The RGB color line images of the RGB LEDs are formed on the CF by the lenticular lens array in the same order as the CF RGB subpixels. The matching of colors between RGB CF subpixels and RGB color light beams contributes to the enhancement of the $\mathrm{CF}$ transmittance by as much as about $300 \%$ theoretically, compared to white-light transmittance. However, there exists the finite acceptance angle $2 \theta_{n}$ (as shown in Fig. 1) that is limited by the finite width of the LLA units, and thus the effective enhancement of transmittance is much lower than the theoretical limit. Increasing the width of the LLA units would be helpful in enhancing the acceptance angle $2 \theta_{n}$, but a large angle may introduce more aberrations in the lenticular lenses, and thus color mismatch and nonuniformity would increase. Thus it is necessary to estimate the optimum condition for best performance of the color-matching LCD in terms of energy efficiency and color uniformity.

\section{RESULTS}

The illuminance and luminance of the assembled prototype 


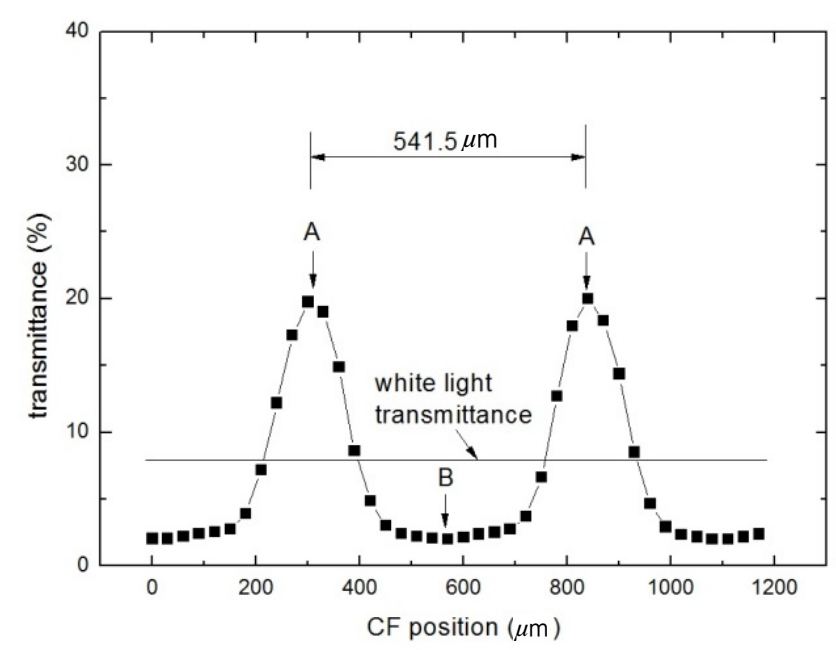

FIG. 5. The change of transmittance in terms of CF position shift. The transmittance for the best color-matching condition (a) is respectively 10.0 and 2.4 times as high as the mismatched transmittance (b) and the average transmittance that can be regarded as white-light transmittance.

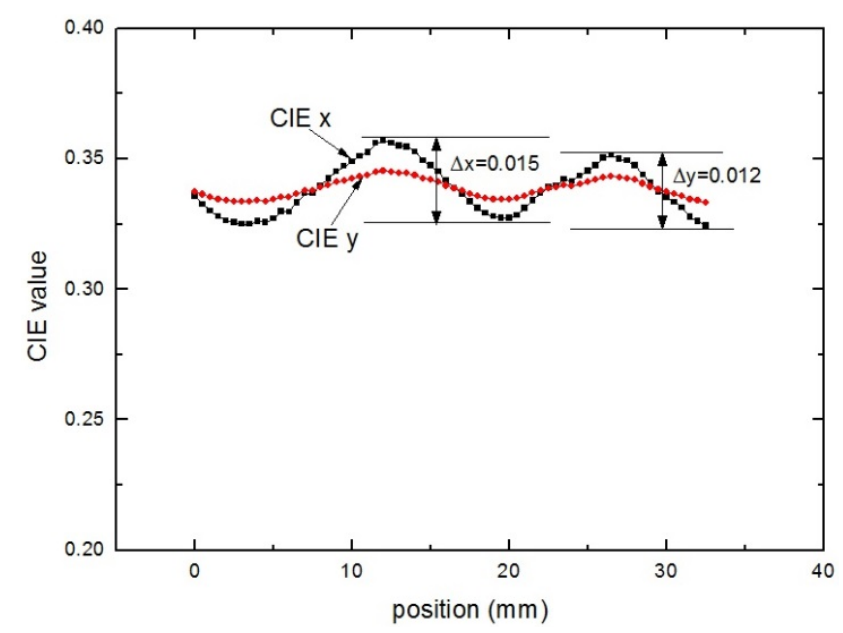

FIG. 6. Profiles of CIE $\Delta x$ and CIE $\Delta y$ values in terms of the position along the $\mathrm{x}$ direction.

color-matching LCD were measured using the T-1 Luxmeter and the CA-210 colorimeter (Konica Minolta). Figure 5 shows the effect of transmittance change due to the $\mathrm{CF}$ position shift. The highest transmittance, designated as A in Fig. 5, is obtained when the color-matching condition is satisfied, and the transmittance becomes very low when the color-matching condition is not satisfied, designated as $\mathrm{B}$ in Fig. 5. The difference between the highest and lowest transmittance values is about a factor of 10 , and the highest transmittance is about 2.4 times the average transmittance that is similar to that of a conventional LCD with uniform white-light backlighting. The slight mismatch between the color filter and the RGB lines is due to the unequal spacings between the RGB color lines that is introduced by the dispersion of the red, green, and blue wavelengths

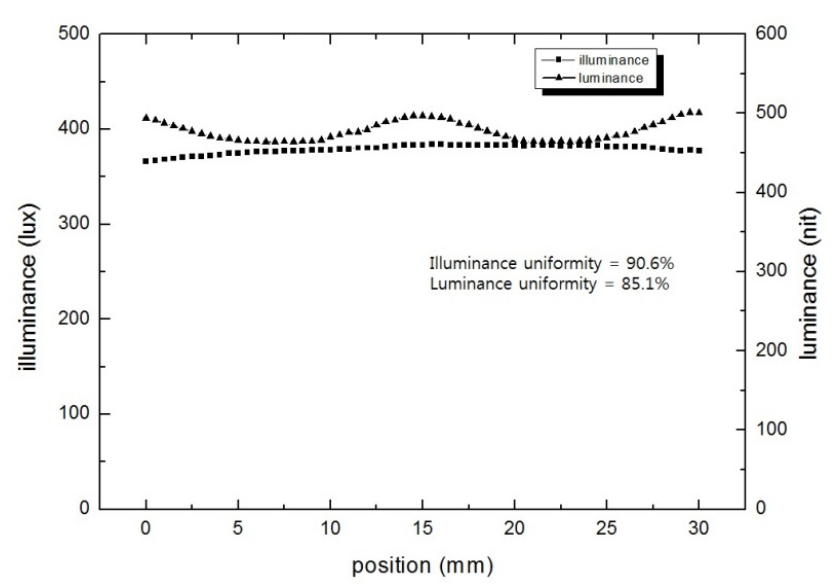

FIG. 7. Profiles of the illuminance and luminance in terms of the position along the $\mathrm{x}$ direction. The illuminance uniformity was $90.6 \%$ and the luminance uniformity was $85.1 \%$.

by the lenticular lens array. This effect is described by Eq. (5). However, since the mismatch is not serious, it is not corrected in our prototype experiment.

The enhancements of luminance and illuminance were $192.5 \%$ and $139.1 \%$ compared to the non-color-matched LCD. These values are very close to the simulation results of $195 \%$ and $146 \%$ for luminance and illuminance respectively. The relatively low increase of the illuminance is due to the limited acceptance angle $2 \theta_{n}=40.16$ degrees, because LED rays falling outside of this angle do not contribute to the transmittance enhancement from colormatching. The relatively large increase of the luminance, as much as $192.5 \%$, comes from the narrowed viewing angle of 81 degrees due to the focusing effect of the lenticular lens array, compared to the conventional viewing angle of 110 degrees. Figure 6 shows the CIE color nonuniformity to be CIE $\Delta x=0.015$ and $\operatorname{CIE} \Delta y=0.012$ centered at $\operatorname{CIE}(x, y)$ $=(0.3255,0.3386)$. The tolerance limit of the color nonuniformity of an LCD is typically 0.01 , a value that human eyes cannot discriminate [11]. The CIE color nonuniformity comes mainly from the separation of RGB LEDs that induces mismatch among the RGB light fluxes. Reducing the separation between the RGB LEDs will lower the CIE color nonuniformity beneath the tolerance limit. In the prototype the minimum available separation was limited by the size of the LEDs currently available. Figure 7 shows the uniformity of the illuminance and luminance in terms of position along the $\mathrm{x}$ direction to be $90.6 \%$ and $85.1 \%$ respectively. The values of the illuminance are independent of the incident ray directions, so its uniformity is much better than that of the luminance, which is dependent on the directions of the incident rays.

\section{DISCUSSION}

In the prototype the RGB color sources were constructed 
from linear arrays of small RGB LEDs. However, a better design for the RGB color sources would be RGB linear lightguides with RGB LEDs located at their ends. Because the human eye is more like a luminance sensor, uniformity in luminance is more important than in illuminance uniformity, and so it is necessary to improve the luminance uniformity in future research. The development of light sources consisting of RGB lightguides instead of LEDs will enable much better uniformity of the luminance and color uniformity with fewer blue LEDs.

The color-matching LCD requires alignment of the RGB LED array, the LLA, and the color filter. The alignment of the RGB LED array with the LLA is less critical because any misalignment of the RGB LED array is reduced due to the demagnification effect of the LLA. However, the alignment of the LLA with the CF is important because otherwise Moire fringes and nonuniformity may occur. The alignment tolerance was not studied in this research because we focused on the feasibility of the color-matching LCD by fabricating a 4-inch sample. Alignment will become critical when the color-matching LCD is larger. A rough estimate of the alignment tolerance would be about $10 \mu \mathrm{m}$ across a $1000 \mathrm{~mm}$ distance that corresponds to a 50-inch display. This alignment tolerance could be attained easily with the current alignment technology used in the LCD industry.

\section{CONCLUSION}

We demonstrated a prototype color-matching LCD comprised of a RGB LED array, an LLA with 8 units, and a color filter. Simulation and experimental results show that this color-matching LCD is a good candidate for an energy-saving LCD. The luminance and illuminance of the color filter were increased as much as $192.5 \%$ and $139.1 \%$ respectively compared to a non-color-matched LCD, due to the color-matching effect. The color-matching technique demonstrated in this prototype LCD will allow us to develop eco-friendly energy-saving displays in the near future.

\section{ACKNOWLEDGMENT}

This work was supported by the 2011 Yeungnam University Research Grant.

\section{REFERENCES}

1. E. Nakasu, Y. Nishida, M. Maeda, M. Kanazawa, K. Mktani, K. Hamasaki, and Y. Nojiri, "Technical development toward implementation of ultra high-definition TV system," SMPTE Mot. Imag. J. 116, 279-286 (2007).

2. X. Yang, Y. Yan, and G. Jin, "Polarized light-guide plate for liquid crystal display," Opt. Express 13, 8349-8356 (2005).

3. Z. Luo, Y.-W. Cheng, and S.-T. Wu, "Polarization-preserving light guide plate for a linearly polarized backlight," J. Display Technol. 10, 208-214 (2014).

4. K.-W. Chien and H.-P. Shieh, "Design and fabrication of an integrated polarized light guide for liquid-crystal-display illumination," Appl. Opt. 43, 1830-1834 (2004).

5. C.-H. Lee, "Angularly positioned LED-based spatial temporal color separation system," Opt. Express 20, 19109-19118 (2012).

6. C.-H. Chen, F.-C. Lin, Y.-T. Hsu, Y.-P. Huang, and H.-P. D. Shieh, "A field sequential color LCD based on color fields arrangement for color breakup and flicker reduction," J. Display Technol. 5, 34-39 (2009).

7. Y.-P. Huang, F.-C. Lin, and H.-P. D. Shieh, "Eco-displays: The color LCD's without color filters and polarizers," J. Display Technol. 7, 630-632 (2011).

8. F.-C. Lin, Y.-P. Huang, C.-M. Wei, and H.-P. D. Shieh, "Color filter-less LCDs in achieving high contrast and low power consumption by stencil field-sequential-color method," J. Display Technol. 6, 98-106 (2010).

9. Y. Taira, H. Numata, D. Nakano, K. Sueoka, F. Yamada, M. Suzuki, M. Noguchi, R. Singh, and E. G. Colgan, "Color filterless liquid crystal display illuminated with LEDs," SID Symposium Digest of Technical Papers 34, 1250-1253 (2003).

10. M.-K. Park, H. J. Lee, J.-S. Park, M. Kim, J. M. Bae, I. Mahmud, and H.-R. Kim, "Design and fabrication of multi-focusing microlens array with different numerical apertures by using thermal reflow method," J. Opt. Soc. Korea 18, 71-77 (2014).

11. R. S. Berns, Principles of Color Technology, Ch. 4 Measuring Colors (John Wiley \& Sons, New York, USA, 2000). 